\title{
Social Connectedness Predicts Lower Loneliness and Depressive Symptoms over Time in Adolescents
}

\author{
Paul E. Jose', Bee Teng Lim² \\ ${ }^{1}$ Victoria University of Wellington, Wellington, New Zealand \\ ${ }^{2}$ University of Otago, Wellington, New Zealand \\ Email: paul.jose@vuw.ac.nz
}

Received 15 June 2014; revised 12 July 2014; accepted 14 August 2014

Copyright (C) 2014 by authors and Scientific Research Publishing Inc.

This work is licensed under the Creative Commons Attribution International License (CC BY). http://creativecommons.org/licenses/by/4.0/

(c) (i) Open Access

\begin{abstract}
Although research has shown that loneliness in adolescents leads to higher rates of depressive symptoms, the presumed protective factor of social connectedness has not been studied in conjunction with these two constructs. The aim of the present study was to determine if social connectedness would predict lower loneliness and depressive symptoms over time in a large sample of adolescents. A large sample of adolescents $(N=1774 ; 9$ - 16 years at Time 1$)$ were surveyed three times, separated by one year each, with self-report measures of social connectedness, loneliness, and depressive symptoms obtained. As expected, social connectedness was negatively associated with loneliness and depressive symptoms concurrently and longitudinally. Contrary to prediction, social connectedness did not function as a buffer between loneliness and depressive symptoms over time. However, consistent with prediction, a significant longitudinal mediation pattern was obtained over the three years: the effect of social connectedness on depressive symptoms was mediated by loneliness. Social connectedness at $\mathrm{T} 1$ predicted a reduction in loneliness at $\mathrm{T} 2$, which in turn predicted a reduction in depressive symptoms at T3. Moderation analyses of this mediation pattern suggested that this obtained mediation result was obtained for males, but not females, and obtained for older adolescents, but not for younger adolescents. The results are congruent with the view that social connectedness exerts an effective protective influence on adolescents against loneliness and depressive symptoms.
\end{abstract}

\section{Keywords}

Social Connectedness, Loneliness, Depressive Symptoms, Adolescence, Longitudinal

How to cite this paper: Jose, P. E., \& Lim, B. T. (2014). Social Connectedness Predicts Lower Loneliness and Depressive Symptoms over Time in Adolescents. Open Journal of Depression, 3, 154-163. 


\section{Introduction}

\subsection{The Relationship between Loneliness and Depressive Symptoms}

Satisfying interpersonal relationships are essential for good physical and mental health. Therefore, it is not surprising that loneliness, defined by Perlman and Peplau (1981) as distress that arises from the perceived discrepancy between desired and achieved social relationships, is associated with increased mortality risk. Compared to socially integrated individuals, people who are lonely have a 50\% increased risk of mortality: Loneliness is a stronger risk factor than excess alcohol use, obesity and lack of physical activities (Holt-Lunstad, Smith, \& Layton, 2010). In terms of mental health, loneliness has long been identified as a risk factor for depressive symptoms in cross-sectional (Eisses et al., 2004; Nolen-Hoeksema \& Ahrens, 2002) and longitudinal studies of older adults (Cacioppo, Hughes, Waite, Hawkley, \& Thisted, 2006; Heikkinen \& Kauppinen, 2004). Specifically, Cacioppo, Hughes et al. (2006) found a bi-directional relationship between loneliness and depressive symptoms in middle-aged and older adults. A pertinent question in relation to these findings is to ask whether levels of loneliness and depression vary across the life span. Although Nolen-Hoeksema and Ahrens (2002) found age group differences in reports of loneliness, with middle-age adults being the loneliest and elderly adults being the least lonely, they reported that the basic association between loneliness and depressive symptoms appeared to be relatively stable across the age-span. In a large nationally representative cross-sectional study of adults aged 18 to 99 years, loneliness and unmet emotional support had the largest and most consistent associations with depressive episodes and symptoms (Barger, Messerli-Burgy, \& Barth, 2014).

Loneliness in adolescence has received considerable attention. It has been argued (Asher \& Paquette, 2003) that loneliness may be a normative experience as adolescents undergo significant changes in social roles, expectations, and identity (Laursen \& Hartl, 2013). Loneliness, despite its prevalence during adolescence, still has the potential to be deleterious. For example, the significant links between loneliness and a wide range of mental health difficulties (e.g., depression, anxiety, suicidal ideation, self-injury, and alcohol and substance misuse) and physical health issues (e.g., poor immune and cardiovascular functioning, sleep deprivation) have been consistently demonstrated in adolescents (Heinrich \& Gullone, 2006; Shevlin, Murphy, Mallet, Stringer, \& Murphy, 2013). Cross-sectional and longitudinal studies have found that loneliness is significantly correlated with depressive symptoms across time in children and adolescents (Hall-Lande, Eisenberg, Christenson, \& NeumarkSztainer, 2007; Lasgaard, Goossens, \& Elklit, 2011; Nangle, Erdley, Newman, Mason, \& Carpenter, 2003; Qualter, Brown, Munn, \& Rotenberg, 2010; Rotenberg et al., 2004; Vanhalst et al., 2012). Mahon et al. (2006) reported that the correlation between loneliness and depressive symptoms in adolescents was in the range of a large effect size ( $r=0.61$ to 0.62$)$.

Although a concurrent, bi-directional link between loneliness and depression is expected by researchers and clinicians, empirical studies looking at the cross-lagged relationships between these two constructs are limited and have yielded mixed findings. Lasgaard et al. (2011) and Rotenberg et al. (2004) found that depressive symptoms predicted subsequent feelings of loneliness in adolescence, but not vice versa. Vanhalst et al. (2012) attributed the lack of consistent cross-temporal associations between loneliness and depression to the short assessment time span (i.e., months or a single year), and they consequently conducted a study examining the reciprocal link across a longer time-span, covering the developmental period from mid- to late-adolescence. Notably, Vanhalst et al. found a reciprocal relationship between loneliness and depressive symptoms from mid- to lateadolescence, with the direction from loneliness to depressive symptoms being stronger than the reverse order. Given these findings, it is clear that adolescence is a critical period in which to study the occurrence of loneliness and depressive symptoms, and to examine how both constructs relate to one another concurrently and over time.

\subsection{Social Connectedness as a Protective Factor}

The essential goal of positive psychology is to move the field of psychology from a focus on psychopathology to a greater consideration of strengths and positive functioning. In recent years, increasing efforts have been directed at identifying protective factors that buffer adolescents against the negative impact of loneliness and depressive symptoms. In their seminal publication, Resnick et al. (1997) reported that family connectedness and school connectedness were powerful negative predictors of adolescents' maladjustment (i.e., emotional distress, suicidal ideation, and substance misuse). Since then, a burgeoning body of research studies has suggested that a 
sense of belongingness, in other words "social connectedness", may act as a buffer against loneliness (Cruwys et al., 2013; Mellor, Stokes, Firth, Hayashi, \& Cummins, 2008). For example, Cruwys et al. (2013) found that social connectedness, measured by the number of social group memberships, was protective against the development of depressive symptoms in a group of non-depressed and initially depressed elderly samples.

Research literature involving studies of adolescents has broadly conceptualised connectedness as a sense of perceived closeness to an individual or group, perceived care and support, a sense of belonging, satisfaction with existing relationships, and ease in talking about problems with significant others (Barber \& Schluterman, 2008). Adolescents' perception of social connectedness has been found to be an important protective factor associated with a reduction in suicidal ideation, suicidal attempts, depressive symptoms, and health-compromising behaviours such as substance misuse, violence involvement, and delinquency (Ackard, Neumark-Sztainer, Story, \& Perry, 2006; Czyz, Liu, \& King, 2012). Libbey, Ireland and Resnick (2002) found in their cross-sectional study negative associations between connectedness and emotional distress, with family connectedness showing the strongest negative relationship, followed by school, neighbourhood, and peer connectedness. In a similar study, Shochet et al. (2006) examined the relationship between school connectedness and mental health difficulties in adolescents across time points: they found that school connectedness negatively correlated with concurrent mental health symptoms, and more importantly, it predicted changes in depressive symptoms, anxiety, and general functioning one year later. Hall-Lande et al. (2007) reported that family connectedness was a very strong protective factor against suicide attempts, particularlyin adolescents who were socially isolated. When gender differences were examined, they found that family connectedness was the only protective factor for suicide attempts in adolescent females, whereas family connectedness, school achievement, and school connectedness buffered adolescent males against the negative impact of loneliness. A study by McGraw et al. (2008) in the same vein found that social connectedness across family, peers, and school domains was inversely associated with depression, anxiety, and stress. Moreover, peer connectedness was a particularly strong predictor of wellbeing. Few studies have examined social connectedness as a buffer of negative affect, however Czyz et al. (2012) found that social connectedness buffered adolescents who had been hospitalised due to acute suicidal ideation and attempts against subsequent depressive symptoms and suicidal ideation.

A paucity exists of longitudinal research that concomitantly examines multiple core aspects of connectedness, namely family, school, peers and community, and their associations with adolescents' wellbeing (Jose, Ryan, \& Pryor, 2012). Jose et al. (2012) found significant positive associations between and among global connectedness, domain-specific connectedness, and psychological wellbeing overtime. Interestingly, they reported that global connectedness predicted wellbeing over time but not the reverse. When individual domains of connectedness were examined, bi-directional relationships between connectedness and wellbeing emerged, particularly for family and school connectedness, suggesting that adolescents had a qualitatively different experience when engaging with schools compared with engaging with peer groups. Drawing their data from the National Longitudinal Study of Adolescent Health, Costello, Swendsen, Rose, and Dierker (2008) found that adolescents who reported greater feelings of connection to parents, peers, and school were more likely to be classified in the no-depressed mood group than either the stable low or early high declining depressed group. The 15-wave (32-year) Dunedin Multidisciplinary Health and Development Study found that adolescents' reports of social connectedness predicted well-being over a decade later, and lack of family connectedness manifested a strong, inverse link with social connectedness in childhood that continued into adolescence, highlighting the enduring significance of positive interpersonal relationship (Olsson, McGee, Nada-Raja, \& Williams, 2013).

\subsection{Aims and Hypotheses}

Our general aim was to investigate the possibility that social connectedness would function as a protective factor against loneliness and depression in adolescence. To achieve this aim, several specific hypotheses were posed:

1) Social connectedness would be found to be negatively correlated with loneliness and depressive symptoms concurrently and longitudinally.

2) Social connectedness would function as a moderator (buffer) of the relationship between loneliness and depressive symptoms.

3) Loneliness would operate as a negative mediator between social connectedness and depressive symptoms longitudinally over three years, i.e., social connectedness would predict a decrease in loneliness over time, and this diminished loneliness would predict lower depressive symptoms in turn. 


\section{Method}

\subsection{Sampling Procedure}

The current study was based on the Youth Connectedness Project dataset (YCP; see Jose et al., 2012). The Youth Connectedness Project obtained data from adolescents by performing a survey three times from 2006 to 2008 , with one year between assessments. The study was designed to investigate the development of social connectedness in adolescence (see Jose et al., 2012), but the survey included a variety of constructs and variables relevant to adolescent development. Adolescents were initially recruited through 78 participating schools in Year 1 from the North Island of New Zealand.

A wide variety of school types (e.g., co-ed and single-sex; middle schools and high schools; religious and state schools) were recruited through a stratified random sampling method and yielded a nationally representative sample of school types. In New Zealand, the "decile" score of a school represents the average socioeconomic status of families contributing children to each school, where 1 indicates the lowest and 10 indicates the highest income brackets. The schools included in this study yielded an average decile of 5.5, thus, the average decile of all schools included in this study was similar to the national average. Participating schools included 61\% urban schools, 33\% suburban schools, and 6\% rural schools, and these frequencies somewhat underrepresented rural schools compared to the national frequencies.

\subsection{Participants}

A total of 1774 adolescents completed the YCP surveys all three years, and were included in the present study. Participants were about evenly distributed across gender (51.9\% female and $48.1 \%$ male), with ages ranging between 9 to 16 years old $(M=12.12, S D=1.73)$ at Time 1 . Participants consisted of 57.7\% New Zealand European, 26.8\% Maori, and 15.3\% from other ethnic backgrounds (i.e., Asian New Zealand and Pacific Island), therefore Maori were somewhat overrepresented in this sample.

\subsection{Measures}

\subsubsection{Social Connectedness}

Previous research (Jose et al., 2012) based on the YCP dataset used here showed that an overall social connectedness construct can be created by combining four related components: family connectedness (11 items), school connectedness (6 items), peer connectedness ( 7 items), and community connectedness (4 items). An example item for family connectedness is: "It means a lot to be a member of my family", for school connectedness: "I like going to school”; for peer connectedness: "My friends and I help each other out”; and for community connectedness: "My family and I can count on our neighbors for help". All items were rated on a 5-point Likert scale, from "Strongly disagree" to "Strongly agree". The social connectedness score was computed by averaging over the 28 items for each of the survey waves. The Cronbach's alphas of social connectedness were excellent for all three years: 87,90 , and 92 respectively.

\subsubsection{Loneliness}

Unfortunately, due to time constraints on the delivery of the survey, a single item represented the loneliness construct: "Please tell us on how many days have you felt these ways in the last week: I felt lonely". Participants answered the item by selecting a point on a 4-point Likert scale, including the options "less than 1 day", “1 - 2 days”, 3 - 4 days” and “5 - 7 days”.

\subsubsection{Depressive Symptoms.}

This construct was assessed with three items taken from the CES-D (Radloff, 1977) using the same 4-point Likert scale as was used with loneliness: "Please tell us on how many days have you felt these ways in the last week: I got upset by things that don't usually upset me; I felt sad; and I could not stop feeling bad, even when others tried to cheer me up”. Adequate internal reliabilities (i.e., Cronbach's alpha) were obtained over time: 0.70, 0.75 and 0.75 respectively.

\subsection{Statistical Analyses}

Missing values constituted only $1 \%$ of the dataset, and a missing values analysis indicated that these values were 
missing completely at random. EM (expectation maximization) imputation (Lin, 2010) was used to estimate and impute this small percentage of missing data.

To test whether loneliness functioned as a longitudinal mediator between social connectedness and the outcome of depressive symptoms, statistical analyses were conducted using the software package Analysis of Moment Structures (AMOS) Ver. 20. A path model with a bootstrap resampling of 3000 iterations and $95 \%$ bias-corrected confidence intervals was performed. This model stipulated social connectedness as the independent variable, loneliness as the mediator, and the construct of depressive symptoms was the single dependent variable.

\section{Results}

\subsection{Descriptive Statistics}

The first hypothesis was evaluated by examining the correlations among the three constructs within given time points and across time points (see Table 1). As predicted, social connectedness was negatively correlated with both loneliness and depressive symptoms within a given time point and across time points, and the latter two constructs were similarly positively correlated. Hypothesis 1 was supported.

\subsection{Would Social Connectedness Moderate the Relationship between Loneliness and Depressive Symptoms over Time?}

Two residualised hierarchical regressions, one for T1-T2 and one for T2-T3, were performed to test the idea embodied in Hypothesis 2 that social connectedness would blunt the positive relationship over time between loneliness and depressive symptoms (for more discussion on how to perform longitudinal moderation see Jose, 2013). For the first time period, the construct of depressive symptoms at $\mathrm{T} 2$ was the dependent variable. In the first step, depressive symptoms at T1 was entered as a predictor, in the second step, loneliness and social connectedness at T1 were entered, and in the third step, the interaction term between loneliness and social connectedness at T1 was entered. The stability coefficient of depressive symptoms proved to be significant, $\beta=0.29, p<0.001$, and the main effect of loneliness yielded statistical significance as well, $\beta=0.15, p<0.001$. The main effect of social connectedness did not yield significance, $\beta=-0.03, p=0.16$, nor did the interaction term, $p=0.93$. The moderation result from T1 to T2 did not support Hypothesis 2, but it did show that loneliness at T1 predicted an increase in depressive symptoms at T2 above and beyond the stability of depressive symptoms.

For the second time period, the construct of depressive symptoms at T3 was the dependent variable. In the first step, depressive symptoms at T2 was entered as a predictor, in the second step, loneliness and social connectedness at T2 were entered, and in the third step, the interaction term between loneliness and social connectedness at $\mathrm{T} 2$ was entered. The stability coefficient of depressive symptoms proved to be significant, $\beta=0.34, p<0.001$, and the two main effects yielded statistical significance as well, loneliness: $\beta=0.07, p<0.05$; and social connectedness: $\beta=-0.07, p<0.01$. As with the T1-T2 time period, the interaction term proved to be non-significant, $p=$ 0.79. The moderation result from T2 to T3 did not support Hypothesis 2 (i.e., the multiplicative model), just as with T1 to T2. However, across the two years, results provided evidence for the additive model for loneliness, and social connectedness contributed variance additively as well from T2 to T3.

\subsection{Did Loneliness Longitudinally Mediate the Impact of Social Connectedness on Depressive Symptoms?}

Hypothesis 3 suggested that social connectedness would indirectly lead to a reduction in depressive symptoms by reducing loneliness. A longitudinal mediation analysis was performed in Amos Ver. 20 (see Figure 1): social connectedness at $\mathrm{T} 1$ was considered the independent variable, residualized loneliness at $\mathrm{T} 2$ was considered the mediator variable, and residualized depressive symptoms at T3 was considered the dependent variable. A bootstrapped analysis involving 3000 resamplings yielded a statistically significant result: $a * b=-0.004$, se $=0.002$, bias corrected $95 \%$ confidence interval $=-0.010$ to $-0.001, p=0.030$. This result provided support for Hypothesis 3.

Additional analyses were conducted to investigate whether this result was equally applicable for the entire adolescent sample, regardless of age and gender. Using equality constraints in Amos, we found that the significant mediation obtained for the whole sample was verified for males, $a * b=-0.006$, se $=0.004$, bias corrected 95\% confidence interval $=-0.016$ to $-0.001, p=0.039$, but not for females, $p=0.21$. A similar analysis on a median 
Table 1. Descriptive statistics.

\begin{tabular}{|c|c|c|c|c|c|c|c|c|c|}
\hline & 1. & 2. & 3. & 4. & 5. & 6. & 7. & 8. & 9. \\
\hline 1) SConn Y1 & & $-0.17^{* * *}$ & $-0.12^{* * * *}$ & $0.66^{* * *}$ & $-0.12^{* * *}$ & $-0.08^{* *}$ & $0.57^{* * *}$ & $-0.15^{* * *}$ & $-0.10^{* * *}$ \\
\hline 2) Lone Y1 & & & $0.55^{* * *}$ & $-0.11^{* * *}$ & $0.28^{* * *}$ & $0.27^{* * *}$ & $-0.12^{* * *}$ & $0.14^{* * *}$ & $0.12^{* * *}$ \\
\hline 3) Depress Y1 & & & & $-0.06^{* *}$ & $0.22^{* * *}$ & $0.29^{* * *}$ & $-0.09^{* *}$ & $0.14^{* * *}$ & $0.21^{* * *}$ \\
\hline 4) SConn Y2 & & & & & $-0.18^{* * *}$ & $-0.15^{* * *}$ & $0.68^{* * *}$ & $-0.17^{* * *}$ & $-0.13^{* * *}$ \\
\hline 5) Lone Y2 & & & & & & $0.62^{* * *}$ & $-0.13^{* * *}$ & $0.24^{* * *}$ & $0.26^{* * *}$ \\
\hline 6) Depress Y2 & & & & & & & $-0.11^{* * *}$ & $0.24^{* * *}$ & $0.34^{* * *}$ \\
\hline 7) SConn Y3 & & & & & & & & $-0.20^{* * *}$ & $-0.18^{* * *}$ \\
\hline 8) Lone Y3 & & & & & & & & & $0.58^{* * *}$ \\
\hline \multicolumn{10}{|l|}{ 9) Depress Y3 } \\
\hline Mean & 3.88 & 1.58 & 1.64 & 3.84 & 1.60 & 1.64 & 3.82 & 1.56 & 1.59 \\
\hline SD & 0.49 & 0.91 & 0.71 & 0.52 & 0.94 & 0.73 & 0.51 & 0.91 & 0.71 \\
\hline
\end{tabular}

Note. SConn = Social connectedness; Lone = Loneliness; Depress = Depressive symptoms. ${ }^{*} p<0.05 ; \stackrel{* *}{p}<0.01 ;{ }^{* * *} p<0.001$.
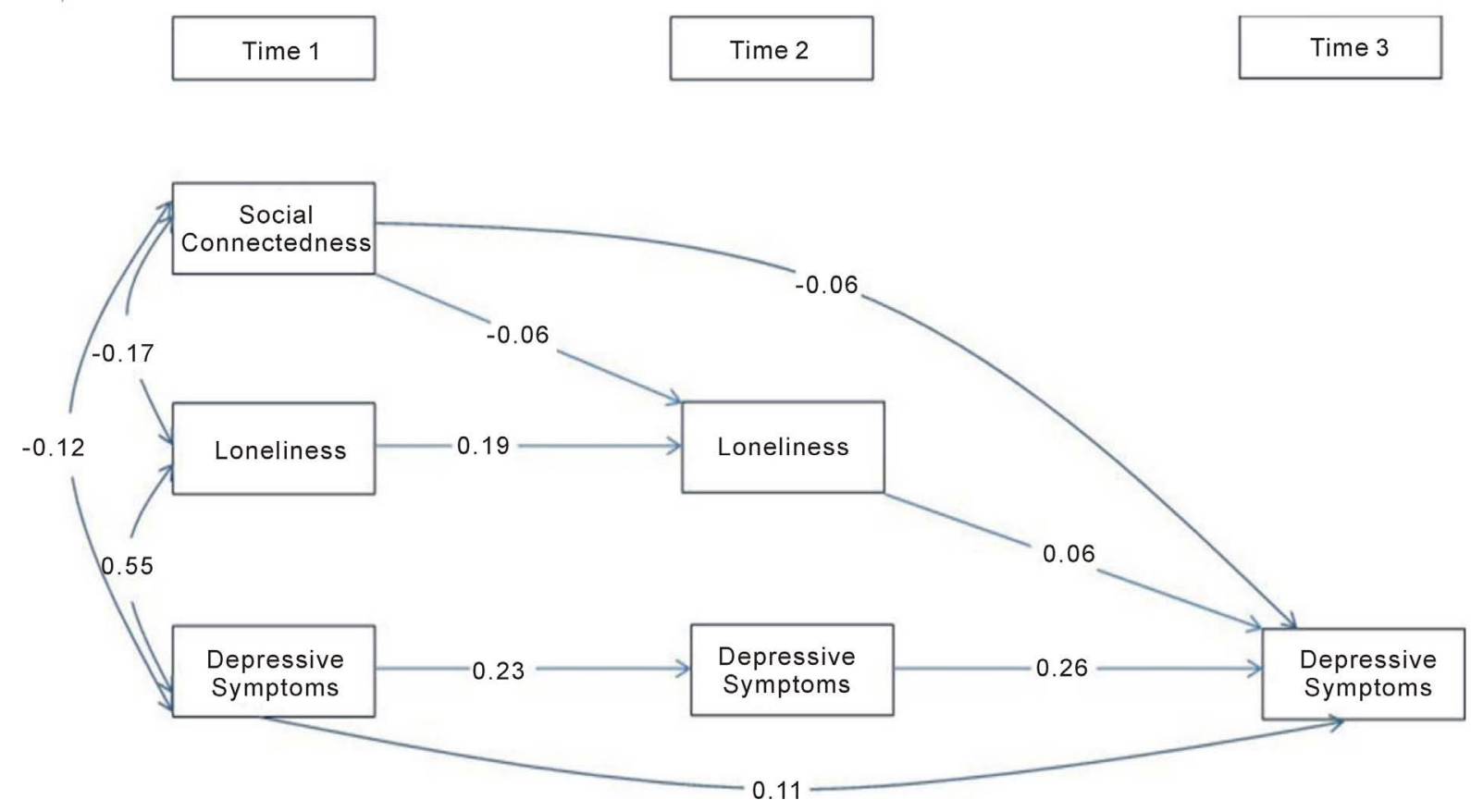

Figure 1. Standardised path model for the direct and indirect effects of social connectedness on depressive symptoms, mediated by loneliness.

split by age indicated that the significant mediation was found for older adolescents (13 - 16 yrs), $a * b=-0.007$, se $=0.005$, bias corrected $95 \%$ confidence interval $=-0.020$ to $-0.001, p=0.05$, but not for younger adolescents ( 9 12 yrs). These results qualify the mediation effect obtained for the entire sample; we can surmise that the obtained mediation result applies to males and to older adolescents.

\section{Discussion}

\subsection{Overview of Findings within the Context of the Literature}

Loneliness has long been found to predict a diverse range of negative physical and psychological outcomes, including poor cardiovascular health, obesity, increased risk of dementia, depressive and anxiety symptoms, as well as alcohol and substance misuse (e.g., Asher \& Paquette, 2003; Barger et al., 2014; Hawkley \& Cacioppo, 2010; Shevlin et al., 2013), but most of the research has focused on adults. Adolescence is identified as a period of life when loneliness is particularly relevant, and there are some theorists who suggest that adolescent experience of 
loneliness may be different from that of children and adults, given developmental changes in identity, autonomy, and individuation as well as social perspective taking in adolescence (Heinrich \& Gullone, 2006; Laursen \& Hartl, 2013). The primary aim of the current study was to investigate social connectedness as a protective factor against loneliness and depression from early to middle adolescence. Longitudinal data were collected over a 3-year period from a large sample of adolescents, and SEM analyses were used to investigate these relationships. More specifically, we predicted that social connectedness would be negatively correlated with loneliness and depressive symptoms concurrently and longitudinally. Our second hypothesis was that social connectedness would function as a moderator (buffer) of the relationship between loneliness and depressive symptoms. And last, we also hypothesised that loneliness would operate as a negative mediator between social connectedness and depressive symptoms longitudinally over three years.

In the first instance, we verified the predicted concurrent and longitudinal associations between loneliness, depressive symptoms, and social connectedness in a national sample of adolescents. Regarding the concurrent relationships, consistent with Cacioppo, Hughes et al. (2006), a significant correlation of a large effect size was found between loneliness and depression $(r=0.55)$. Previous findings of social connectedness being negatively correlated with loneliness and depression concurrently have also been reported by several authors (e.g., Libbey et al., 2002). Longitudinal studies examining the link between multiple aspects of connectedness and adolescents wellbeing are still lacking, but existing literature (Costello et al., 2008; Hall-Lande et al., 2007; Jose et al., 2012; McGraw et al., 2008; Olsson et al., 2013) has identified a positive impact of social connectedness on adolescent's loneliness and depression. In the present study, we found, in line with the findings from other longitudinal studies, significant longitudinal correlations between social connectedness, loneliness and depression in the expected directions.

Much of the literature has examined the extent to which risk factors such as negative reactivity, low levels of social engagement, negative cognitive style, low self-esteem, and low trust beliefs in others predispose adolescents to experience increasing level of loneliness (e.g., Qualter et al., 2013). However, moderators of the influence of loneliness on depression have not been identified or studied. To fill this gap, we predicted that social connectedness would function as a moderator that buffers adolescents from the positive relationship between loneliness and depressive symptoms that has been identified by many researchers. Contrary to our prediction, social connectedness did not act as a moderator (buffer) between loneliness and depressive symptoms over time. The analysis for the first time period (T1 to T2), however, showed that loneliness predicted a subsequent increase in depressive symptoms above and beyond the temporal stability of depressive symptoms. For the second time period, as with the first time period, our moderational hypothesis was not supported, but we found evidence for an additive model for loneliness, with social connectedness explaining a significant amount of change in depressive symptomsover time. To our knowledge, the moderating role of social connectedness on the link between loneliness and depression has not been examined in previous studies. While the null result may be surprising, the lack of a significant moderational impact observed in the current study could be due to the strong correlations observed between loneliness and depression. Baron and Kenny (1986) suggested that moderating variables are typically introduced when an unexpectedly weak or inconsistent relationship is observed between a predictor and a criterion variable. On the other hand, mediation is more suitable when there is a strong relationship between the predictor and criterion variable, and that relationship was explored next.

Our third hypothesis concerned whether social connectedness would indirectly lead to a reduction in subsequent depressive symptoms by decreasing loneliness. A significant longitudinal mediation finding was observed, with loneliness acting as a longitudinal mediator between social connectedness and depressive symptoms in our three-wave study of adolescents' wellbeing. In addition, when age and gender differences were examined, we found that the mediational relationship remained significant for males (but not females) and older adolescents aged 13 to 16 years (but not younger adolescents). The question of whether relationships among adolescents' loneliness and depressive symptoms are moderated by gender is interesting and germane. Although adolescent females tend to self-report higher levels of loneliness and depressive symptoms, some studies report that the strengths of the association between loneliness and depressive symptoms are similar for boys and girls (Lasgaard, Goossens, \& Elklit, 2011; Olsson et al., 2013; Vanhalst et al., 2012). In their studies with the elderly, Cacioppo, Hughes et al. (2006) found that the association between loneliness and depressive symptoms was strong and significant for both males and females, but the association was significantly stronger for males. Given their findings, they suggested that the cause of depressive symptoms might differ for males and females, with loneliness as a more frequent or powerful cause of depressive symptoms for males than females. Literature is lacking on how 
to explain why loneliness would function as a significant mediator between social connectedness and depressive symptoms, and further work needs to tackle this intriguing finding.

\subsection{Contributions and Limitations}

Our dataset possessed several clear strengths. The longitudinal nature of the data (i.e., three waves across three years) allowed for the examination of intra-individual changeover time. In addition, the combination of risk and protective factors offers a more complex view of the lives of adolescents compared to research that only focuses on the negative aspects. The inclusion of social connectedness as a protective factor suggests potential pathways for healthy development and strategies for prevention and intervention. In addition, the large, diverse population-based sample used in the current study yields results that may be more generalisable to the larger adolescent and young adult population.

Despite these strong points, the present study is not without weaknesses. Our study was limited by the measurement of loneliness with a single item. Loneliness has a different meaning in different domains of relationships (Goossens et al., 2009); the single item ("I felt lonely") may not provide a comprehensive picture of the feelings of loneliness in adolescents. To capture a more complete experience of chronic social isolation, additional information such as more detailed information of the quality and quantity of various domains of interpersonal friendships would be beneficial. Furthermore, corroborative data from external sources such as peer ratings, parent ratings, or teacher ratings could lend further validity to the assessment of loneliness. For example, Lasgaard, Goosens, Bramsen et al. (2011) found that peer- and family-related loneliness were associated with depression, anxiety, and suicidal ideation, whereas peer-related and romantic loneliness were associated with social phobia. Consequently, assessing loneliness using a unidimensional approach may obscure the complicated relationships between various forms of loneliness (Heinrich \& Gullone, 2006; Shevlin, Murphy, \& Murphy, 2014), thus decreasing the ability to identify individuals at risk for loneliness. In the same vein, we combined four distinct domains of connectedness mathematically into a single score of global connectedness. The global construct provides an overview of the nature of connectedness (i.e., adolescents' ability to establish and maintain meaningful relationships across multiple domains), but we acknowledge that the domain view can also be helpful, given that different patterns of relationships have been observed when social connectedness was measured at a global compared to a domain level (e.g., Jose et al., 2012).

\section{Conclusion}

In summary, our study provides support that loneliness was associated with significantly higher reports of depressive symptoms, while global social connectedness was inversely related to adolescents' loneliness and depressive symptoms. Although we did not find that social connectedness was a significant moderator of the basic loneliness to depression relationship, both loneliness and social connectedness additively explained depressive symptoms in adolescents. The key finding of the present study was the result that social connectedness indirectly predicted lowered depressive symptoms by decreasing loneliness. This current study highlights the need for intervention research aimed at helping adolescents who experience chronic loneliness. Parents and school counsellors should be attentive to signs of chronic loneliness in their children or students before it evolves into serious bouts of depression (Vanhalst et al., 2012). Prevention programs that emphasise the promotion of family connectedness and school-based interventions that facilitate the development and maintenance of meaningful relationships over time, offer great potential in reducing adolescent's social isolation and are likely to yield longterm psychological benefits (Hall-Lande et al., 2007; Jose et al., 2012).

\section{References}

Ackard, D. M., Neumark-Sztainer, D., Story, M., \& Perry, C. (2006). Parent-Child Connectedness and Behavioural and Emotional Health among Adolescents. American Journal of Preventive Medicine, 30, 59-66. http://dx.doi.org/10.1016/j.amepre.2005.09.013

Asher, S. R., \& Paquette, J. A. (2003). Loneliness and Peer Relations in Childhood. Current Directions in Psychological Science, 12, 75-78. http://dx.doi.org/10.1111/1467-8721.01233

Barber, B. K., \& Schluterman, J. M. (2008). Connectedness in the Lives of Children and Adolescents: A Call for Greater Conceptual Clarity. Journal of Adolescent Health, 43, 209-216. http://dx.doi.org/10.1016/j.jadohealth.2008.01.012 
Barger, S. D., Messerli-Burgy, N., \& Barth, J. (2014). Social Relationship Correlates of Major Depressive Disorder and Depressive Symptoms in Switzerland: Nationally Representative Cross-Sectional Study. BMC Public Health, 14, $273-282$. http://dx.doi.org/10.1186/1471-2458-14-273

Baron, R. M., \& Kenny, D. A. (1986). The Moderator-Mediator Variable Distinction in Social Psychological Research: Conceptual, Strategic and Statistical Considerations. Journal of Personality and Social Psychology, 51, 1173-1182. http://dx.doi.org/10.1037/0022-3514.51.6.1173

Cacioppo, J. T., Hughes, M. E., Waite, L. J., Hawkley, L. C., \& Thisted, R. A. (2006). Loneliness as a Specific Risk Factor for Depressive Symptoms: Cross-Sectional and Longitudinal Analyses. Psychology and Aging, 21, 140-151. http://dx.doi.org/10.1037/0882-7974.21.1.140

Costello, D. M., Swendsen, J., Rose, J. S., \& Dierker, L. C. (2008). Risk and Protective Factors Associated with Trajectories of Depressed Mood from Adolescence to Early Adulthood. Journal of Consulting and Clinical Psychology, 76, 173-183. http://dx.doi.org/10.1037/0022-006X.76.2.173

Cruwys, T., Dingle, G. A., Haslam, C., Haslam, S. A., Jetten, J., \& Morton, T. A. (2013). Social Group Memberships Protect against Future Depression, Alleviate Depression Symptoms and Prevent Depression Relapse. Social Science and Medicine, 98, 179-186. http://dx.doi.org/10.1016/j.socscimed.2013.09.013

Czyz, E. K., Liu, Z., \& King, C. A. (2012). Social Connectedness and One-Year Trajectories among Suicidal Adolescents Following Psychiatric Hospitalisation. Journal of Clinical Child and Adolescent Psychology, 41, 214-226. http://dx.doi.org/10.1080/15374416.2012.651998

Eisses, A. M. H., Kluiter, H., Jongenelis, K., Pot, A. M., Beekman, A. T. F., \& Ormel, J. (2004). Risk Indicators of Depression in Residential Homes. International Journal of Geriatric Psychiatry, 19, 634-640. http://dx.doi.org/10.1002/gps.1137

Goossens, L., Lasgaard, M., Luyckx, K., Vanhalst, J., Mathias, S., \& Masy, E. (2009). Loneliness and Solitude in Adolescence: A Confirmatory Factor Analysis of Alternative Models. Personality and Individual Differences, 47, 890-894. http://dx.doi.org/10.1016/j.paid.2009.07.011

Hall-Lande, J. A., Eisenberg, M. E., Christenson, S. L., \& Neumark-Sztainer, D. (2007). Social Isolation, Psychological Health, and Protective Factors in Adolescence. Adolescence, 42, 265-286.

Hawkley, L. C., \& Cacioppo, J. T. (2010). Loneliness Matters: A Theoretical and Empirical Review of Consequences and Mechanisms. Annals of Behavioural Medicine, 40, 218-227. http://dx.doi.org/10.1007/s12160-010-9210-8

Heikkinen, R., \& Kauppinen, M. (2004). Depressive Symptoms in Late Life: A 10-Year Follow-Up. Archives of Gerontology and Geriatrics, 38, 239-250. http://dx.doi.org/10.1016/j.archger.2003.10.004

Heinrich, L., \& Gullone, E. (2006). The Clinical Significance of Loneliness: A Literature Review. Clinical Psychology Review, 26, 695-718. http://dx.doi.org/10.1016/j.cpr.2006.04.002

Holt-Lunstad, J., Smith, T. B., \& Layton, J. B. (2010). Social Relationships and Mortality Risk: A Meta-Analytic Review. PLoS Medicine, 7, e1000316. http://dx.doi.org/10.1371/journal.pmed.1000316

Jose, P. E. (2013). Doing Statistical Mediation and Moderation. New York: The Guilford Press.

Jose, P. E., Ryan, N., \& Pryor, J. (2012). Does Social Connectedness Promote a Greater Sense of Well-Being in Adolescence over Time? Journal of Research on Adolescence, 22, 235-251. http://dx.doi.org/10.1111/j.1532-7795.2012.00783.x

Lasgaard, M., Goossens, L., Bramsen, R. H., Trillingsgaard, T., \& Elklit, A. (2011). Different Sources of Loneliness Are Associated with Different Forms of Psychopathology in Adolescence. Journal of Research in Personality, 45, $233-237$. http://dx.doi.org/10.1016/j.jrp.2010.12.005

Lasgaard, M., Goossens, L., \& Elklit, A. (2011). Loneliness, Depressive Symptomatology, and Suicide Ideation in Adolescence: Cross-Sectional and Longitudinal Analyses. Journal of Abnormal Child Psychology, 39, 137-150. http://dx.doi.org/10.1007/s10802-010-9442-x

Laursen, B., \& Hartl, A. C. (2013). Understanding Loneliness during Adolescence: Developmental Changes That Increase the Risk of Perceived Social Isolation. Journal of Adolescence, 36, 1261-1268. http://dx.doi.org/10.1016/j.adolescence.2013.06.003

Libbey, H., Ireland, M., \& Resnick, M. (2002). Social Connectedness: Is Protection Cumulative? Journal of Adolescent Health, 30, 102. http://dx.doi.org/10.1016/S1054-139X(01)00366-4

Lin, T. H. (2010). A Comparison of Multiple Imputation with EM Algorithm and MCMC Method for Quality of Life Missing Data. Quality and Quantity, 44, 277-287. http://dx.doi.org/10.1007/s11135-008-9196-5

Mahon, N. E., Yarcheski, A., Yarcheski, T. J., Cannella, B. L., \& Hanks, M. M. (2006). A Meta-Analytic Study of Predictors of Loneliness during Adolescence. Nursing Research, 55, 308-315. http://dx.doi.org/10.1097/00006199-200609000-00003

McGraw, K., Moore, S., Fuller, A., \& Bates, G. (2008). Family, Peer, and School Connectedness in Final Year Secondary School Students. Australian Psychologist, 43, 22-37. http://dx.doi.org/10.1080/00050060701668637 
Mellor, D., Stokes, M., Firth, L., Hayashi, Y., \& Cummins, R. (2008). Need for Belonging, Relationship Satisfaction, Loneliness and Life Satisfaction. Personality and Individual Differences, 45, 213-218. http://dx.doi.org/10.1016/j.paid.2008.03.020

Nangle, D. W., Erdley, C. A., Newman, J. E., Mason, C. A., \& Carpenter, E. M. (2003). Popularity, Friendship Quantity and Friendship Quality: Interactive Influences on Children's Loneliness and Depression. Journal of Clinical Child and Adolescent Psychology, 32, 546-555. http://dx.doi.org/10.1207/S15374424JCCP3204_7

Nolen-Hoeksema, S., \& Ahrens, C. (2002). Age Differences and Similarities in the Correlates of Depressive Symptoms. Psychology and Aging, 17, 116-124. http://dx.doi.org/10.1037/0882-7974.17.1.116

Olsson, C. A., McGee, R., Nada-Raja, S., \& Williams, S. (2013). A 32-Year Longitudinal Study of Child and Adolescent Pathways to Wellbeing in Adulthood. Journal of Happiness Studies, 14, 1069-1083. http://dx.doi.org/10.1007/s10902-012-9369-8

Perlman, D., \& Peplau, L. A. (1981). Toward a Social Psychology of Loneliness. In R. Gilmour, \& S. Duck (Eds.), Personal Relationships: 3. Relationships in Disorder (pp. 31-56). London: Academic Press.

Qualter, P., Brown, S. L., Munn, P., \& Rotenberg, K. J. (2010). Childhood Loneliness as a Predictor of Adolescent Depressive Symptoms: An 8-Year Longitudinal Study. European Child and Adolescent Psychiatry, 19, 493-501. http://dx.doi.org/10.1007/s00787-009-0059-y

Qualter, P., Brown, S. L., Rotenberg, K. J., Vanhalst, J., Harris, R. A., \& Goosens, L. (2013). Trajectories of Loneliness during Childhood and Adolescence: Predictors and Health Outcomes. Journal of Adolescence, 36, 1283-1293. http://dx.doi.org/10.1016/j.adolescence.2013.01.005

Radloff, L. S. (1977). The CES-D Scale: A Self-Report Depression Scale for Research in the General Population. Applied Psychological Measurement, 1, 385-401. http://dx.doi.org/10.1177/014662167700100306

Resnick, M. D., Bearman, P. S., Blum, R. W., Bauman, K. E., Harris, K. M., Jones, J. et al. (1997). Protecting Adolescents from Harm: Findings from the National Longitudinal Study on Adolescent Health. Journal of the American Medical Association, 278, 823-832. http://dx.doi.org/10.1001/jama.1997.03550100049038

Rotenberg, K. J., McDougall, P., Boulton, M. J., Vaillancourt, T., Fox, C., \& Hymel, S. (2004). Cross-Sectional and Longitudinal Relations among Peer-Reported Trustworthiness, Social Relationships, and Psychological Adjustment in Children and Early Adolescents from the United Kingdom and Canada. Journal of Experimental Child Psychology, 88, 46-67. http://dx.doi.org/10.1016/j.jecp.2004.01.005

Shevlin, M., Murphy, J., Mallet, J., Stringer, M., \& Murphy, J. (2013). Adolescent Loneliness and Psychiatric Morbidity in Northern Ireland. British Journal of Clinical Psychology, 52, 230-234. http://dx.doi.org/10.1111/bjc.12018

Shevlin, M., Murphy, S., \& Murphy, J. (2014). Adolescent Loneliness and Psychiatric Morbidity in the General Population: Identifying “at Risk” Group Using Latent Class Analysis. Nordic Journal of Psychiatry. http://dx.doi.org/10.3109/08039488.2014.907342

Shochet, I. M., Dadds, M. R., Ham, D., \& Montague, R. (2006). School Connectedness in an Underemphazised Parameter in Adolescent Mental Health: Results of a Community Prediction Study. Journal of Clinical Child and Adolescent Psychology, 35, 170-179. http://dx.doi.org/10.1207/s15374424jccp3502_1

Vanhalst, J., Klimstra, T. A., Luyckx, K., Scholte, R. H. J., Engels, R. C. M. E., \& Goossens, L. (2012). The Interplay of Loneliness and Depressive Symptoms across Adolescence: Exploring the Role of Personality Traits. Journal of Youth and Adolescence, 41, 776-787. http://dx.doi.org/10.1007/s10964-011-9726-7 
Scientific Research Publishing (SCIRP) is one of the largest Open Access journal publishers. It is currently publishing more than 200 open access, online, peer-reviewed journals covering a wide range of academic disciplines. SCIRP serves the worldwide academic communities and contributes to the progress and application of science with its publication.

Other selected journals from SCIRP are listed as below. Submit your manuscript to us via either submit@scirp.org or Online Submission Portal.
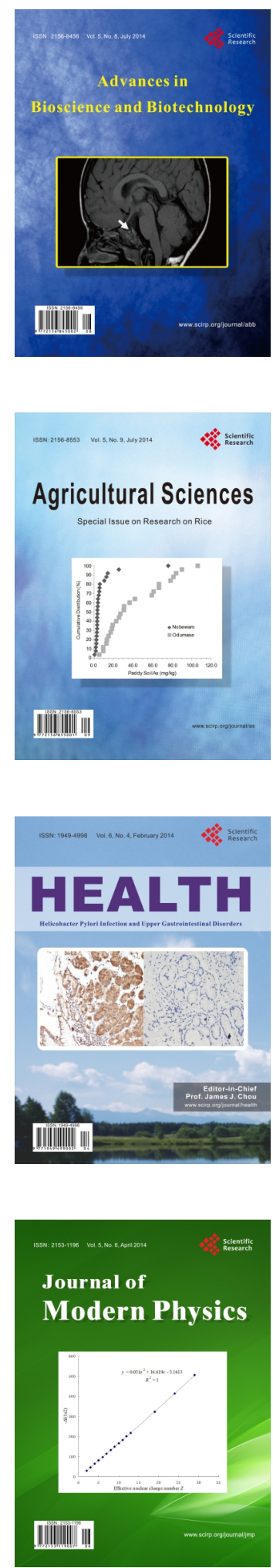
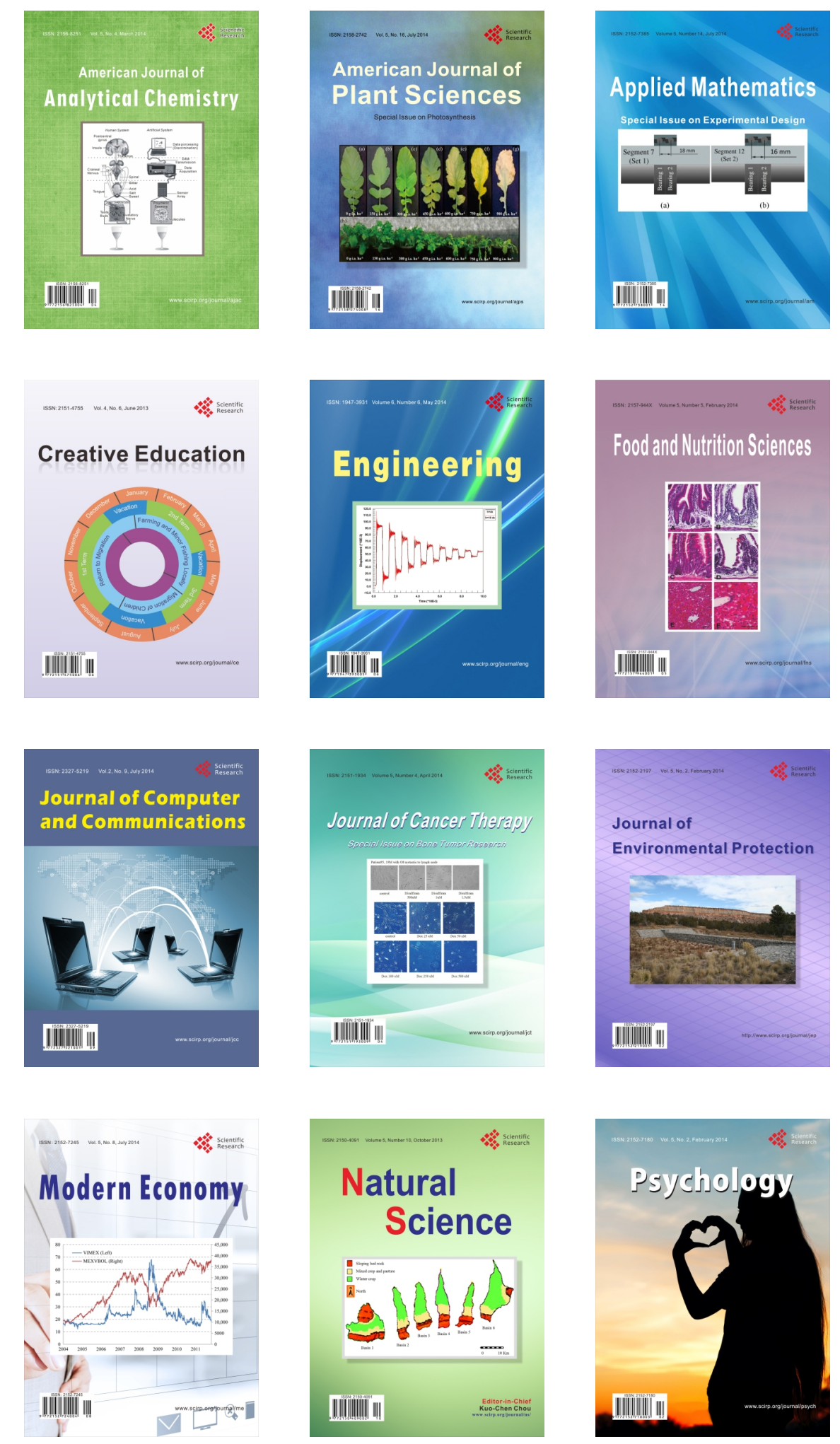